\title{
OPEN Verloren negatively regulates the expression of IMD pathway dependent antimicrobial peptides in Drosophila
}

\author{
Pragya Prakash ${ }^{1}$, Arghyashree Roychowdhury-Sinha ${ }^{1}$ \& Akira Goto ${ }^{1,2 \bowtie}$
}

Drosophila immune deficiency (IMD) pathway is similar to the human tumor necrosis factor receptor (TNFR) signaling pathway and is preferentially activated by Gram-negative bacterial infection. Recent studies highlighted the importance of IMD pathway regulation as it is tightly controlled by numbers of negative regulators at multiple levels. Here, we report a new negative regulator of the IMD pathway, Verloren (Velo). Silencing of Velo led to constitutive expression of the IMD pathway dependent antimicrobial peptides (AMPs), and Escherichia coli stimulation further enhanced the AMP expression. Epistatic analysis indicated that Velo knock-down mediated AMP upregulation is dependent on the canonical members of the IMD pathway. The immune fluorescent study using overexpression constructs revealed that Velo resides both in the nucleus and cytoplasm, but the majority $(\sim 75 \%)$ is localized in the nucleus. We also observed from in vivo analysis that Velo knock-down flies exhibit significant upregulation of the AMP expression and reduced bacterial load. Survival experiments showed that Velo knock-down flies have a short lifespan and are susceptible to the infection of pathogenic Gram-negative bacteria, $P$. aeruginosa. Taken together, these data suggest that Velo is an additional new negative regulator of the IMD pathway, possibly acting in both the nucleus and cytoplasm.

Innate immunity provides a potent host defense against microbial infections. A plethora of genetic tools, less genetic redundancy, and most importantly the lack of adaptive immunity and a high degree of evolutionary conservation to mammals have made the fruit fly Drosophila melanogaster an excellent model organism to decipher the fundamental molecular mechanism of innate immunity ${ }^{1-5}$. The Drosophila innate immunity is mainly composed of two systems, namely cellular and humoral immune responses. The cellular immune reaction is mediated by insect blood cells called hemocytes involving in various cellular aspects such as phagocytosis, encapsulation, melanization, etc. The humoral response is characterized by the challenge-induced transcription of hundreds of immune response effectors, including antimicrobial peptides (AMPs), predominantly in the fat body (an insect equivalent of the mammalian liver), the hemocytes, and barrier epithelia, namely in the gut and the tracheal systems. The AMPs are positively charged amphipathic small peptides. They are classically known to eliminate invading microorganisms generally by using two mechanisms: (1) membrane disruption and 2) inhibition of essential cellular functions $s^{6-9}$. However, recent findings revealed that the function of AMPs is not only restricted to microbe killing but also extended to other biological aspects such as gut homeostasis, neurology, and tumor control ${ }^{10}$. There are two distinct innate immune signaling pathways known in Drosophila, namely the Toll and immune deficiency (IMD) pathways ${ }^{11,12}$. They share similarity to mammalian Toll-like receptor signaling pathway and tumor necrosis factor a receptor (TNFR) signaling pathway, respectively and control the expression of respective AMP readouts such as Drosomycin and Diptericin ${ }^{13,14}$. The Toll pathway is mainly activated by fungal and/or Gram-positive bacteria and mediated by several factors such as Toll receptor, Drosophila myeloid differentiation primary response $88(\mathrm{dMyD} 88)^{15}$, tube and pelle kinase ${ }^{16}$, and dorsal related immunity factor (Dif) ${ }^{17}$. Sensing and recognition of pathogen-associated molecular patterns (PAMPs) and of the so-called danger signals in the Toll pathway are mediated through secreted receptors such as $\beta$-glucan binding protein 1 and 3 (GNBP-1, -3), peptidoglycan recognition protein short-type A (PGRP-SA) ${ }^{18-20}$, and Psh ${ }^{21,22}$. In contrast, the IMD pathway is preferentially activated by Gram-negative bacteria and initiated by the direct binding of the

${ }^{1}$ INSERM, Université de Strasbourg, CNRS, Insect Models of Innate Immunity (M3I; UPR9022), 67084 Strasbourg, France. ${ }^{2}$ Sino-French Hoffmann Institute, School of Basic Medical Science, Guangzhou Medical University, Guangzhou 511436, China. ${ }^{\circledR}$ email: goto@unistra.fr 
diaminopimelic acid-type peptidoglycan (DAP-type PGN), which is common to most Gram-negative bacteria, to the peptidoglycan recognition protein long-type C (PGRP-LC) and PGRP-LE receptors ${ }^{23-25}$. Mechanistically, this ligand-receptor binding induces the adaptor complex composed of death domain containing IMD ${ }^{12,26}$, Fas-associated death domain (FADD) ${ }^{27,28}$, and Death related ced-3/Nedd2-like caspase (DREDD) ${ }^{29}$. DREDD becomes activated with ubiquitination by Death-associated inhibitor of apoptosis 2 (DIAP2) ${ }^{30-32}$. Activated

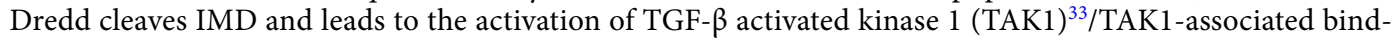
ing protein $2(\mathrm{TAB} 2)^{31}$ complex. The dTAK1/dTAB2 complex is responsible to activate the IKK signalosome composing of dIKK $\beta$ (ird5) ${ }^{34}$ and dIKK $\gamma(\mathrm{key})^{35}$, which in turn cleaves the NF- $\kappa B$ protein Relish. The activated transcription factor Relish is then delivered into the nucleus and triggers hundreds of effectors, including AMPs such as Diptericin ${ }^{34,36,37}$.

Flies deficient for the above mentioned positive regulators lead to compromised immune response, resulting in bacterial overload and high susceptibility to infection ${ }^{13,14}$. Notably, flies deficient for negative regulators also die quickly in association with enhanced activation of the IMD pathway ${ }^{38,39}$. It is generally considered that an uncontrolled immune system leads to detrimental effects in the host, but the underlying mechanism remains elusive. In the IMD pathway, several negative regulators have been identified. PGRP-SC and PGRP-LB amidases degrade PGN into non-stimulatory fragments outside of the cell ${ }^{40,41}$. PGRP-LF interacts with PGRP-LC and produces inactive PGRP-LF/PGRP-LC heterodimer ${ }^{42}$. Inside the cell, Pirk interacts with IMD to disrupt the receptor complex ${ }^{43-45}$. Interestingly, Ragab et al. further discovered that the induction of Pirk occurs through the activation of PVR/Ras-MAPK pathway ${ }^{46}$. The ubiquitin-specific protease, dUSP36/Scny, and deubiquitinating enzyme, faf, are involved in the degradation of ubiquitinated IMD ${ }^{47,48}$. The E3 ubiquitin ligase, Plenty of SH3s (POSH), was shown to poly-ubiquitinate TAK1 for the proteasomal degradation ${ }^{49}$. Trabid and Cylindromatosis (CYLD) deubiquitinases bind to dTAK $1^{50}$ and dIKK $\gamma(\text { key })^{51,52}$, respectively and modulate K63-linked polyubiquitination of their respective target. Ubiquitin binding protein dRYBP was proposed to function for the degradation of Relish together with $\mathrm{SKPA}^{53}$. As such, a number of negative regulators were shown to participate in the process of ubiquitination/de-ubiquitination in the IMD pathway.

To further advance the knowledge, Fukuyama et al. undertook a pathway-wide and time-lapse proteomic analysis using 11 canonical components of the IMD pathway and identified $\sim 400$ interacting proteins ${ }^{54}$. Following RNAi-mediated knock-down experiment, more than half of the candidates show a dysregulated expression of the Attacin A-luciferase reporter, proving that the system is potent enough to identify promising candidates. Gene ontology analysis of candidates revealed a significant signature of "small ubiquitin-like modifier (SUMO) binding" as one of the important molecular functions. Indeed, flies heterozygote for the gene encoding E2 SUMO conjugating enzyme, DmUbc9 (lesswright mutant: lwr), were highly susceptible to Escherichia coli (E. coli) infection. The $l w r$ flies had an increased bacterial burden and showed a reduced expression of Attacin A, a representative AMP of the IMD pathway. Further biochemical experiments provided evidence that one of the components of the IMD pathway namely dIKK $\beta$ is SUMOylated upon heat-killed E. coli stimulation. These results suggest that the SUMOylation plays a critical role in the activation of the IMD pathway ${ }^{54}$.

In this study, we focused on Verloren (Velo), which encodes a SUMO-specific protease and was originally identified from the IMD pathway interactome. We first observed that silencing of Velo led to upregulation of the AMP expression both in S2 cells and adult flies. Epistasis analysis showed that this AMP upregulation by knocking down of Velo depends on the canonical components of the IMD pathway, however, overexpression of Velo did not repress the IMD pathway. Cellular localization study indicated that the majority of Velo is localized in the nucleus, but some are also in the cytoplasm. Velo knock-down flies also showed upregulation of the AMP expression, but they had reduced lifespan and were susceptible to pathogenic Gram-negative bacteria P. aeruginosa infection. Taken together, we propose that Velo is a new negative regulator, which controls the expression of IMD pathway dependent AMPs.

\section{Materials and methods}

Fly strains. Stocks were raised on standard cornmeal-yeast-agar medium ( $63 \mathrm{~g}$ of cornmeal, $11 \mathrm{~g}$ of yeast powder, $4.8 \mathrm{~g}$ of agar, $47 \mathrm{~g}$ of sugar and $4.2 \mathrm{~g}$ of methylhydroxyl -4 benzoate per litter) at $25^{\circ} \mathrm{C}$ with $60 \%$ relative humidity. Transgenic flies for Velo knock-down (VDRC ID: 18004-GD and 103524-KK; carrying a UAS-RNAi against Verloren) and $d I K K \beta$ (ird5) knock-down (VDRC ID: 26427; carrying a UAS-RNAi against $d I K K \beta$ (ird5)) were obtained from the Vienna Drosophila RNAi Center (VDRC). As controls, we used flies carrying UAS-RNAi transgene against GFP (397-05) obtained from Kyoto stock center (DGRC) or VDRC (VDRC ID: 60100). Rel$i s h^{E 20}$ and $D i f^{\prime m c 55}$ were used as a mutant deficient for the IMD and Toll pathway, respectively. Flies carrying Gal4 drivers, ubiquitous daughterless ( $\mathrm{da}$-Gal4) and the fat body-specific (c564-Gal4), were obtained from Bloomington Drosophila Stock Center (Bloomington, USA).

Microbial strains and infections. The entomopathogenic fungi B. bassiana was used to perform natural fungal infection ${ }^{18}$. Pathogenic Gram-negative bacterial strain P. aeruginosa ${ }^{56}$ was used for septic injuries. The survival experiment was performed by pricking the flies with a sharp tungsten needle dipped in the concentration of $\mathrm{OD}_{600}=0.5$ bacterial solution. Sterile injury with PBS was used as a control. After the infection, the survival of the flies was monitored over 11 days at $25^{\circ} \mathrm{C}$.

Colony forming unit (CFU) assay. Flies were pricked with a concentrated kanamycin-resistant E. coli MC4100 strain carrying the pBB2:GFP plasmid (a gift from Dr. Eleonora García Véscovi) or $\mathrm{OD}_{600}=1.0$ P. aeruginosa solution. After $6 \mathrm{~h}$ or $24 \mathrm{~h}$ incubation of the flies at $29^{\circ} \mathrm{C}$, a total of 5 flies per sample with at least 8 biologi- 
cal replicates for each genotype were homogenized in $150 \mu \mathrm{l}$ of LB medium, serially diluted, and plated onto the kanamycin containing LB agar plates. The next day, numbers of colonies were counted to calculate CFU per fly.

Quantitative RT-qPCR. For quantitative expression analysis of Diptericin, Attacin, Metchnikowin, Cecropin, Drosomycin, and Velo, total RNAs from the flies were isolated by the standard protocol using a TRIzol Reagent RT bromoanisole solution (MRC). Briefly, $1 \mu \mathrm{g}$ of total RNA was reverse transcribed using iScript TM cDNA synthesis Kit (Biorad). Real-time PCR was performed using $100 \mathrm{ng}$ of cDNAs in 384-well plates on

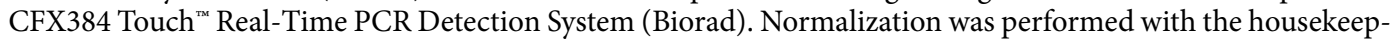
ing gene Ribosomal protein 49 (Rp49). The qPCR data were analyzed by the $\Delta \Delta C T$ method. Sequences of RTqPCR primers are shown in Supplementary Table 1.

Synthesis of double-stranded (ds) RNAs. The PCR fragments with two T7 promoter sequences at both ends were amplified by PCR and used for the templates for dsRNA production. Fragments for each gene were as follows: GFP (nt 26-302, GenBank L29345), Velo (nt 1540-1881 and 1933-2266, NCBI NM_139799), Imd (nt 146-490, NCBI NM_133166), PGRP-LC (nt 365-620, NCBI NM_001169925), Relish (nt 848-1107, NCBI NM_057746). dsRNAs were synthesized by in vitro transcription with T7 MEGAscript T7 transcription kit (AM1334; Ambion). Two independent dsRNAs for Velo were generated to eliminate the possibility of an offtarget effect. dsRNA against GFP $(d s G F P)$ was used as a negative control.

Cell culture and transfection. Schneider 2 (S2) cells were cultured at $25^{\circ} \mathrm{C}$ in Schneider's medium (Biowest) supplemented with $10 \%$ fetal calf serum (FCS) and $8 \mathrm{mM}$ penicillin/streptomycin (Gibco). For transient transfection, a total of $0.6 \times 10^{6}$ cells were seeded per well in a 24 -well plate. Transfection was performed by the calcium phosphate co-precipitation method. Each plate was co-transfected with $1 \mu \mathrm{g}$ of indicated tagged overexpression plasmids (pMT-short Velo-V5, pAC-long Velo-WT-HA ${ }^{57}$, pAC-long Velo-CS-HA ${ }^{57}$, pAC-PGRP-LC $(\mathrm{TM}+\mathrm{Intra})-\mathrm{V} 5^{58}$, or pAC-Relish $\left.\Delta \mathrm{S} 29-\mathrm{S} 45^{36}\right), 50 \mathrm{ng}$ of AttacinA-firefly luciferase reporter, $10 \mathrm{ng}$ of Actin5Crenilla luciferase transfection control reporter, and each dsRNAs $(2.0 \mu \mathrm{g} / \mathrm{well})$. After $12-16 \mathrm{~h}$ of the transfection, the cells were washed with PBS and incubated in a fresh medium with or without $500 \mu \mathrm{M} \mathrm{CuSO}_{4}$. The next day, cells were stimulated with heat-killed $E$. coli for $24 \mathrm{~h}$ with the multiplicity of infection $(\mathrm{MOI})=40$. Firefly and Renilla luciferase activities of the cell lysate were measured by a dual luciferase assay kit (Promega).

Immunoprecipitation and western blot. After transfection of the indicated plasmids at $72 \mathrm{~h}$, the cells were harvested, washed by PBS, and lysed in lysis buffer containing a complete protease inhibitor cocktail (Roche). Immunoprecipitation was performed overnight with rotation at $4{ }^{\circ} \mathrm{C}$, using monoclonal anti-V5 antibody coupled to agarose beads (Sigma, A7345). Proteins from immune-precipitates and total cell lysates were separated by SDS-PAGE and detected by western blotting using rabbit anti-HA (1;3000; Abcam, ab9110)/mouse anti-HA (1:3000; Roche, 12CA5, 11583816001), rabbit anti-V5 (1:3000; Abcam, ab9116), and/or mouse antiactin (1:10,000; Millipore, clone C4, MAB1501R) antibodies.

Immunofluorescence. Cells were seeded on 8-wells Lab-Tek Chamber Slide, washed with PBS, and fixed with $2 \%$ paraformaldehyde. Cells were then permeabilized with $0.1 \%$ Triton X-100 and blocked with bovine serum albumin (BSA). After blocking, samples were incubated with mouse anti-HA (1:500) or mouse antiV5 (1:500) antibody overnight at $4{ }^{\circ} \mathrm{C}$. After washing cells, Alexa 488 anti-mouse (Thermo Fischer Scientific, A28175) was used for the secondary antibody reaction with 1:500 dilution. Slides were mounted in Vectashield/ DAPI solution and samples were imaged using a Zeiss LSM780 confocal microscope. Images were subsequently processed using ImageJ or Photoshop software.

Statistical analysis. Unpaired two-tailed Student's $t$ test was used for statistical analysis of data with GraphPad Prism (GraphPad Software). Error bars indicate standard deviation. Survival curves were plotted and analyed by log-rank analysis (Kaplan-Meier method) using GraphPad Prism (GraphPad Software). $p$ values lower than 0.05 were considered statistically significant.

\section{Results}

Velo knock-down upregulates expression of the IMD pathway regulated AMPs. Our preliminary RNAi screen of the IMD pathway interactome candidates indicated Verloren (Velo, CG10107), a putative SUMO specific protease, as a negative regulator of the IMD pathway. We first confirmed by using two independent dsRNAs that Velo is significantly knocked down (Fig. 1A). We next monitored activation of the IMD pathway by using Attacin-luciferase reporter in the absence or presence of heat-killed E. coli (HKE). Consistent with the preliminary RNAi screen result, the knock-down of Velo resulted in upregulation of Attacin-luciferase activity both in the absence and presence of HKE stimulation (Fig. 1B). We observed a similar upregulation of endogenous AMPs, namely Attacin, Diptericin, and Metchnikowin (Fig. 1C-E), further confirming that Velo knock-down triggers the induction of the IMD pathway dependent antimicrobial peptide expression at both basal and immune-stimulated conditions.

Upregulation of AMP expression by Velo knock-down is dependent on the canonical components of the IMD pathway. To investigate the cellular target of Velo in the IMD pathway, we next performed the epistatic analysis by using double knock-down of Velo and the canonical components of the IMD pathway (Fig. 2). We chose three major components of the pathway, namely PGRP-LC (recognition), IMD 
A

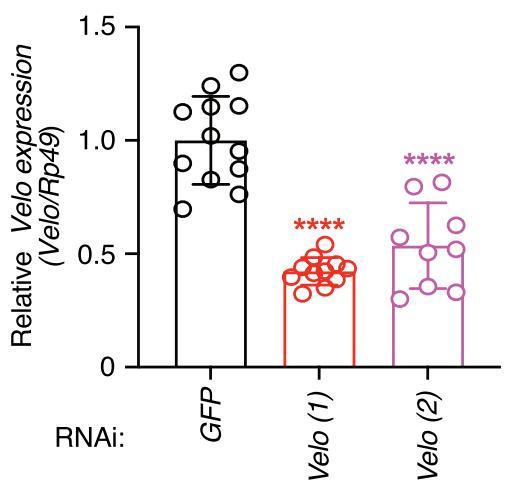

C
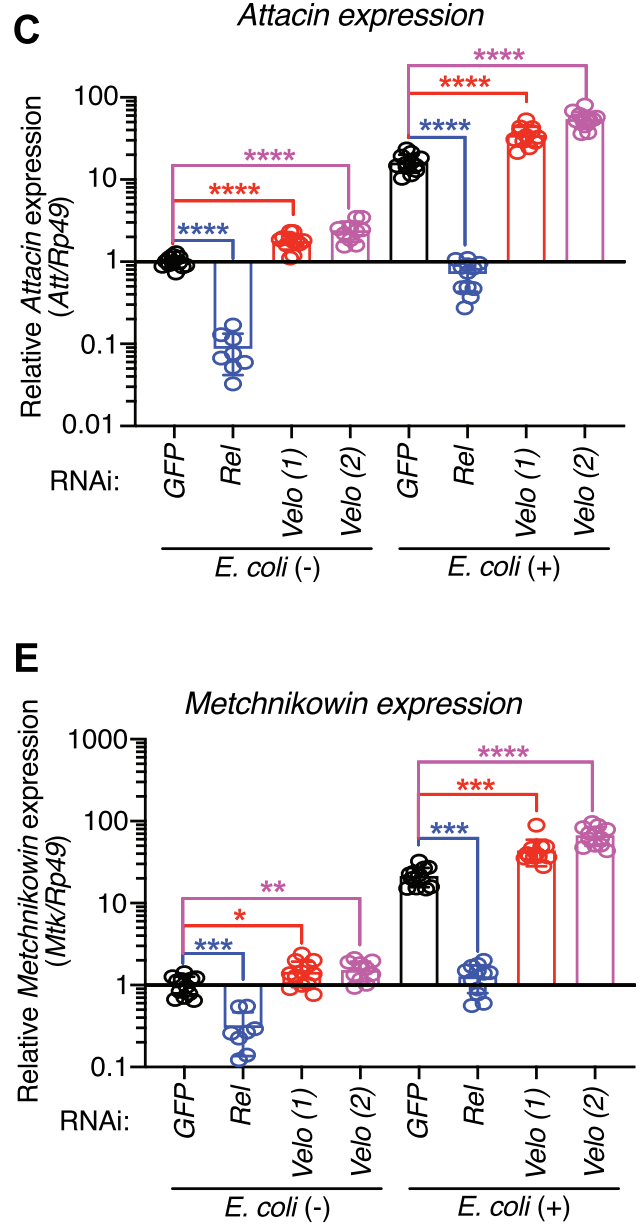

B

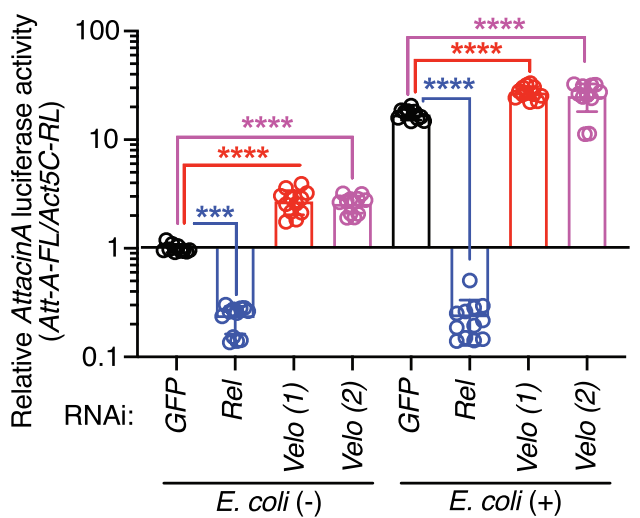

D Diptericin expression

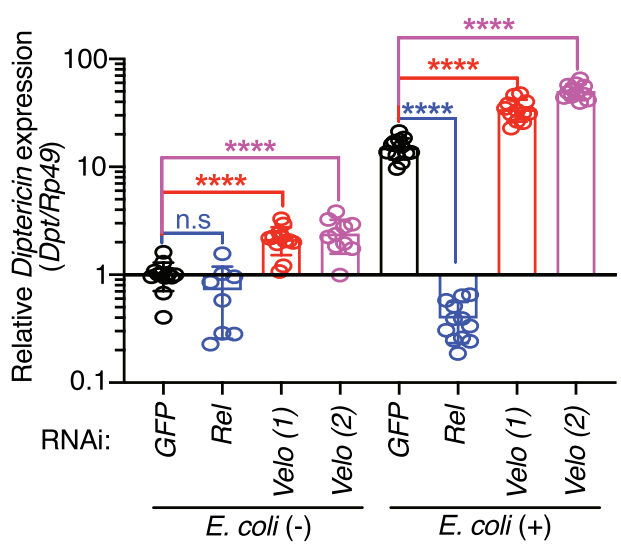

Figure 1. Upregulation of the IMD pathway-regulated AMPs in Velo knock-down cells. (A) Two dsRNAs targeting to different regions of Velo transcript (designated as Velo(1) and Velo(2)) were synthesized to detect a possible off-target effect. dsRNA against GFP was used as a negative control. Three days after the dsRNA transfection into S2 cells, total RNAs were extracted from the cells and Velo expression was monitored by RT-qPCR. Expression of the Ribosomal protein 49 (Rp49) was used as the internal control for normalization. (B) S2 cells were transfected with the indicated dsRNA together with the AttacinA-luciferase (Att-A-FL) and the transfection control Actin5C-Renilla luciferase (Act5C-RL) reporters. After stimulation with heat-killed E. coli (40 bacteria/cell), the relative AttacinA-luciferase activity was calculated based on the ratio of Att-A-FL/Act5C-RL. The value of control GFP knock-down cells was set as 1. dsRNAs against GFP and Relish (Rel) were used as a negative or positive control, respectively. (C-E) Same as in (B), S2 cells were transfected with the indicated dsRNAs, and endogenous expression level of antimicrobial peptides (AMPs) namely Attacin (C), Diptericin (D), and Metchnikowin (E) was monitored by RT-qPCR. Expression of the Rp49 was used as the internal control for normalization. The data points were collected from three independent experiments. Each experiment includes at least two bio-replicates. Student's $t$ test was used for statistical analysis: ${ }^{*} p<0.05,{ }^{* *} p<0.01,{ }^{* * *} p<0.001,{ }^{* * *} p<0.0001$. n.s. indicates statistically non-significant. 
A

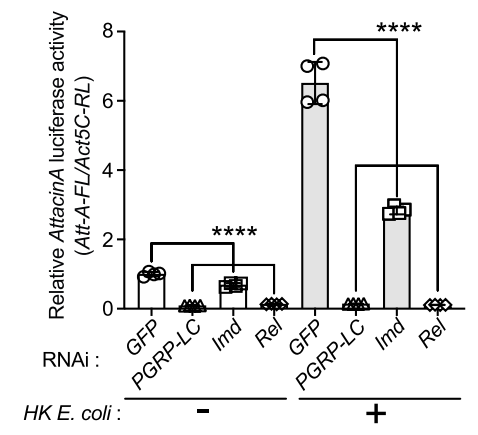

C

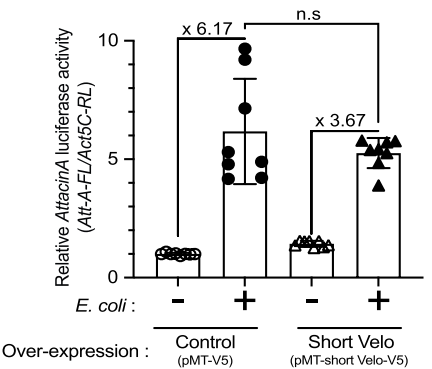

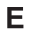

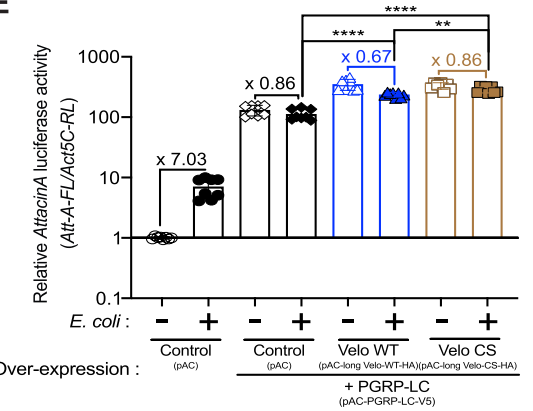

B

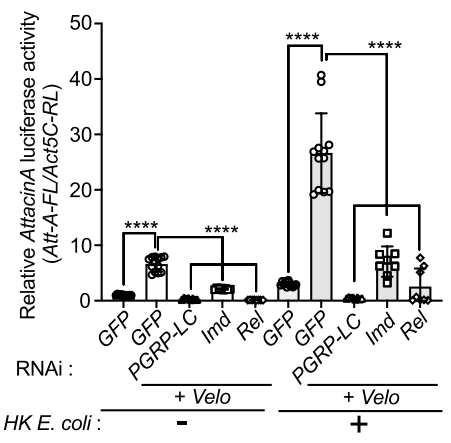

D

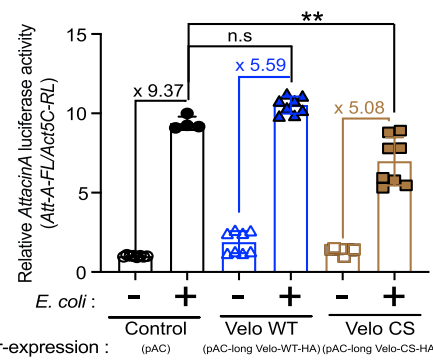

$\mathbf{F}$

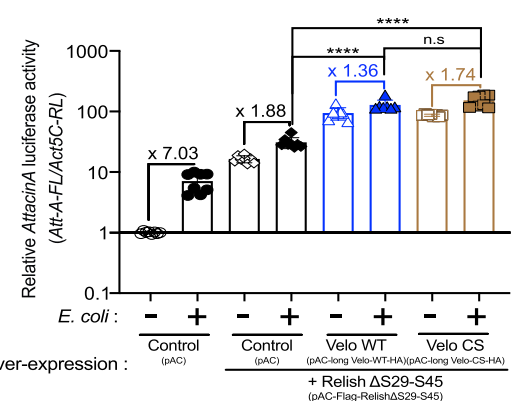

G

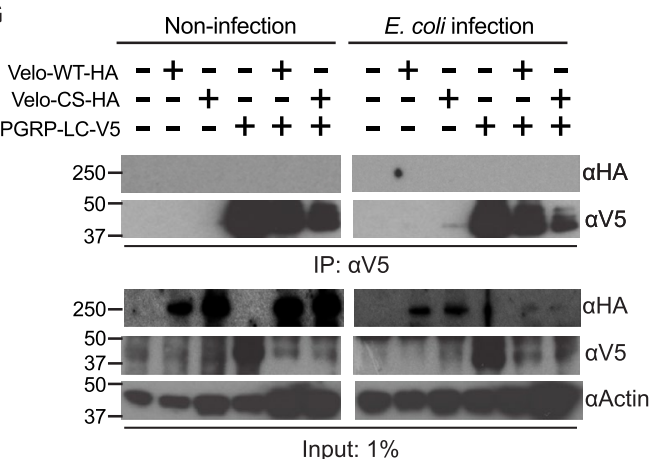

Figure 2. Epistatic analyses of Velo in the IMD pathway. (A,B) PGRP-LC, IMD, or Rel, was knocked down individually (A) or together with Velo (B). After the indicated dsRNA transfection, the activation of the IMD pathway was monitored by AttacinA-luciferase activity $(A t t-A-F L / A c t 5 C-R L)$ in the absence or presence of heatkilled E. coli stimulation. The value of GFP knock-down control cells was set as 1. (C,D) After transfection of the plasmids encoding for a short-form (Short Velo) (C) or long-form of Velos (either wild-type "Velo WT" or protease-dead "Velo CS") (D), the AttacinA-luciferase activity was measured in the absence or presence of heatkilled E. coli stimulation. (E,F) Long-form of Velo (Velo WT-HA or Velo CS-HA) was co-expressed either with PGRP-LC (E) or Relish (F), and the AttacinA-luciferase activity was measured. The data points were collected from two independent experiments. Each experiment includes at least two bio-replicates. Student's $t$-test was used for statistical analysis: ${ }^{* *} p<0.01,{ }^{* * * *} p<0.0001$. n.s. indicates statistically non-significant. (G) S2 cells were co-transfected with plasmids encoding for the tagged PGRP-LC (PGRP-LC-V5) and long-form of Velo (VeloWT-HA or Velo-CS-HA). After stimulation of the transfected cells by heat-killed E. coli, the cell lysates were co-immunoprecipitated (IP) and immunoblotted by the indicated antibodies. Empty vector transfection and single overexpression of PGRP-LC or Velo were used as controls. $1 \%$ of the volume of cell lysate was used as input. 
(adaptor), or Relish (transcription factor). We first confirmed that the knock-down of each gene significantly represses Attacin-luciferase reporter activation (Fig. 2A). We then examined the effect of Velo by double knockdown experiment. The result showed that constitutive activation of AttacinA-luciferase activity by Velo knockdown was significantly repressed in all three gene knock-down conditions. This repression was also observed in the presence of HKE stimulation (Fig. 2B). Of note, a similar result was observed when other components of the pathway namely DIAP2, TAB2, or IKK $\beta$ was knocked down (Supplementary Fig. 1).

We next investigated if overexpression of Velo modulates the IMD pathway signaling. There are two isoforms of Velo, namely short-form (711 aa) and long-form (1,833 aa). The short-form lacks the N-terminal Glutaminerich region. We constructed copper-inducible pMT plasmid expressing a V5-tagged short-form of Velo and used HA-tagged long-forms of Velo, namely Velo-WT (wild-type) and Velo-CS (point mutation from cysteine to serine at amino acid position 1,624) (gift from Pr. Liqun Luo) ${ }^{57}$. We confirmed by RT-qPCR that Velo is overexpressed ( $\sim 600$-fold for the short-form and $\sim 100$-fold for the long-form) (Supplementary Fig. 2A,B). However, we did not observe any significant effect on the induction of Attacin-luciferase activity (Fig. 2C,D). A similar result was noted for the endogenous expression of Attacin and Cecropin (Supplementary Fig. 2C-F). We also tried to overexpress Velo in the context of PGRP-LC or Relish overexpression where the IMD pathway becomes constitutively active. In both cases, we did not observe any significant repression of the Attacin-luciferase activity (Fig. 2E,F).

Although the overexpression of Velo did not impact the IMD pathway, the result of double knock-down partially suggested that Velo may act at parallel or upstream of PGRP-LC (Fig. 2B). We, therefore, tested the interaction between Velo and PGRP-LC by co-immunoprecipitation assay. While PGRP-LC-V5 was detected with an anti-V5 antibody, the band corresponding to Velo in the complex was not detected by the anti-HA antibody (Fig. 2G). Therefore, we did not observe the interaction between PGRP-LC and Velo in our experimental condition.

Velo is mostly localized in the nucleus but some are in the cytoplasm. We confirmed by western blot that tagged short- and long-form of Velos (short Velo-V5 and long Velo-HA) are expressed at the expected size, $\sim 81-\mathrm{kDa}$ and $\sim 204-\mathrm{kDa}$, respectively (Fig. 3A). We next examined the cellular localization of Velo by immune cell staining. The result showed that both forms are localized in the nucleus and cytoplasm (Fig. 3B). After counting the numbers of stained cells, we noted that the majority of Velo localizes in the nucleus $(\sim 75 \%)$. In addition, we found that the short-form is more diffused into the cytoplasm $(\sim 25.0 \%$ of Velo short-form localizes in the cytoplasm, whereas it was $\sim 17.7 \%$ for the long Velo-WT) (Fig. 3C and see discussion). The cytoplasmic localization of long Velo-CS was similar to that of long Velo-WT $(\sim 17.6 \%)$. Although we do not exclude the possibility that ectopically overexpressed Velo modifies the subcellular localization, these results suggest that the majority of Velo especially the long-form is localized in the nucleus (see also in "Discussion").

Effect of Velo knock-down in in vivo adult flies. To investigate the in vivo function of Velo, we generated Velo knock-down flies by crossing the UAS-Velo-RNAi line with ubiquitous daughterless (da)-Gal4 driver (hereafter referred to as $d a>$ Velo-RNAi flies). We first noted that many of $d a>V e l o$-RNAi flies died out during larval/pupal stages, pointing that strong knock-down of Velo induces a developmental defect as previously reported $^{57}$. Consistently, $d a>$ Velo RNAi adult escapers showed a marginal 50\% knock-down efficiency (Fig. 4A) and led to the demise after 17 days (Fig. 4B). Nonetheless, $d a>$ Velo RNAi escapers showed an elevated level of Diptericin expression at both basal and E. coli stimulated conditions (Fig. 4C). To examine the direct impact of the injected bacteria, we performed a colony formation unit (CFU) assay. The result showed that $d a>V e l o-R N A i$ escapers reduced injected E. coli burden (Fig. 4E). Interestingly, the expression of the Toll pathway readout namely Drosomycin was unaffected upon the infection of Micrococcus luteus (Fig. 4D). These results indicate that Velo is specifically required for the downregulation of the IMD pathway both in vitro and in vivo.

Velo knock-down flies are susceptible to the infection of Gram-negative bacteria $P$. aeruginosa but not to fungi $B$. bassiana. Partial lethality at larval/pupal stage and short lifespan of $d a>$ Velo-RNAi adult escapers made it difficult to pursue investigating the in vivo function of Velo in response to microbial infections. We, therefore, generated flies which has Velo knocked down only in major innate immune organs, namely the fat body using the c564-Gal4 driver. We confirmed that Velo is significantly knocked down in two different lines of c564> Velo-RNAi flies (designated as GD and KK) (Fig. 5A). The lifespan experiment showed that both lines had a shorter lifespan as compared to control RNAi flies (Fig. 5B). Nevertheless, c564 > Velo-RNAi flies survived far longer than $d a>$ Velo-RNAi escapers (45 days for $c 564>$ Velo-RNAi vs 17 days for $d a>$ Velo-RNAi) and they did not show any compromised survival effect at least until 15 days after emerging. We, therefore, used $\sim 3$ to 5 day-old $c 564>$ Velo-RNAi flies in the following infection experiment. As shown in Fig. 5C,D, RT-qPCR result showed that the expression level of the IMD pathway-regulated AMPs, namely Diptericin and Attacin, was significantly increased upon infection of P. aeruginosa, whereas Drosomycin and Puckered expressions were unaffected (Fig. 5E, Supplementary Fig. 3). Consistent with Fig. 4E, CFU assay indicated that c564>Velo-RNAi flies had a reduced $P$. aeruginosa burden (Fig. 5F). Nevertheless, c564 $>$ Velo-RNAi flies were highly susceptible to $P$. aeruginosa infection (Fig. 5G). In contrast, they did not show any obvious effect after the infection of $B$. 


\section{A}
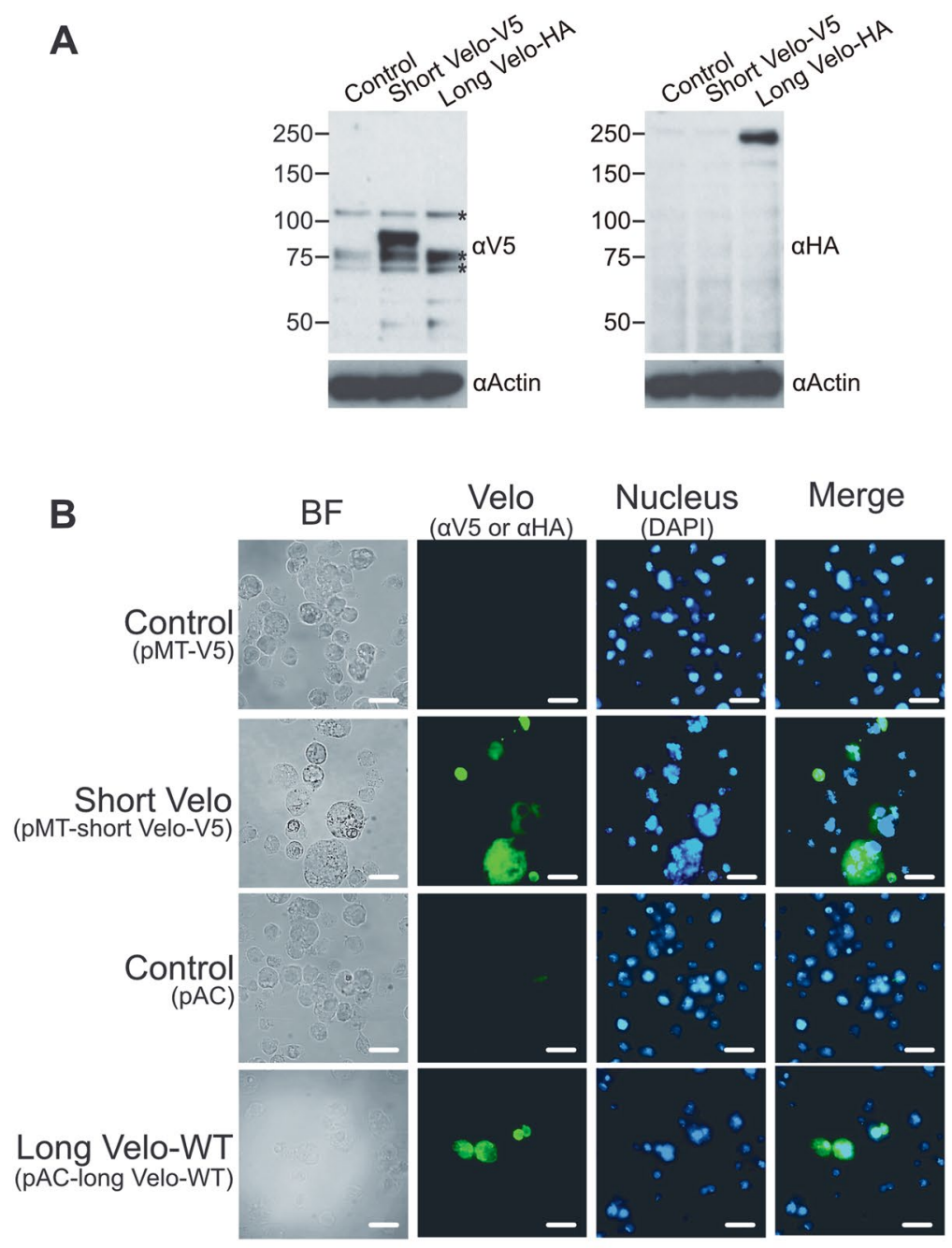

C

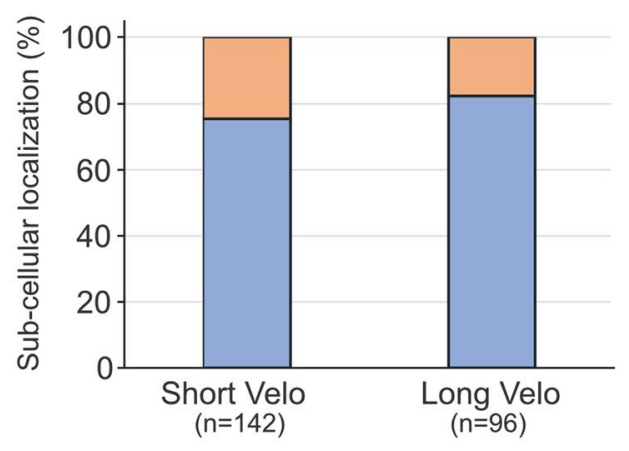

Nucleus

Nucleus + Cytoplasm

Figure 3. Velo localizes mostly in the nucleus but also in the cytosol. (A) S2 cells were transfected with the plasmid expressing either short- or long-form of Velo (designated as Short Velo and Long Velo-WT, respectively). For Short Velo in which Velo cDNA is inserted in the metallothionein driven pMT vector, the transfected cells were harvested $24 \mathrm{~h}$ after the $\mathrm{CuSO}_{4}$ induction. For Long Velo-WT constructed in Actin5C-promoter driven pAC vector, the cells were collected $48 \mathrm{~h}$ after the transfection. Cell lysates were immunoblotted with indicated antibodies. Detection of actin was used as an internal loading control. Asterisks indicate non-specific bands, which were detected in control empty vector transfected cells (mock). (B) After the transfection, the cells were stained by anti-V5 (for short Velo) or anti-HA antibody (for long Velo-WT) and revealed by Alexa488 (green) secondary antibody. DAPI (blue) was used to stain the nucleus. Bar indicates $10 \mu \mathrm{m}$. (C) The numbers of stained cells indicated were analyzed for the subcellular localization of Velo either in the nucleus or the nucleus + cytoplasm. 
A
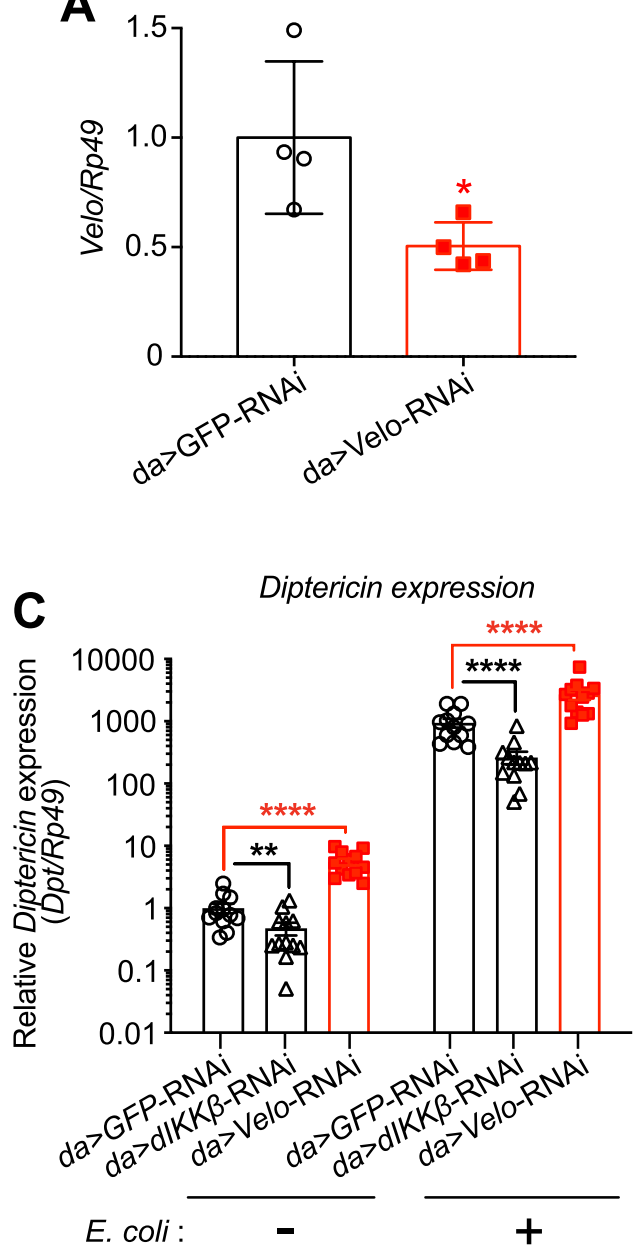

B

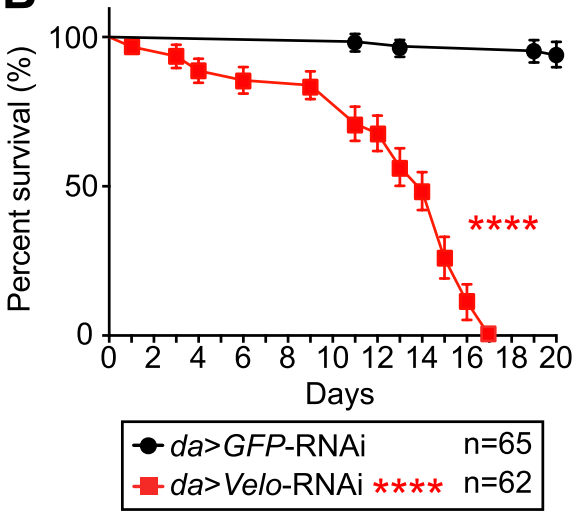

D

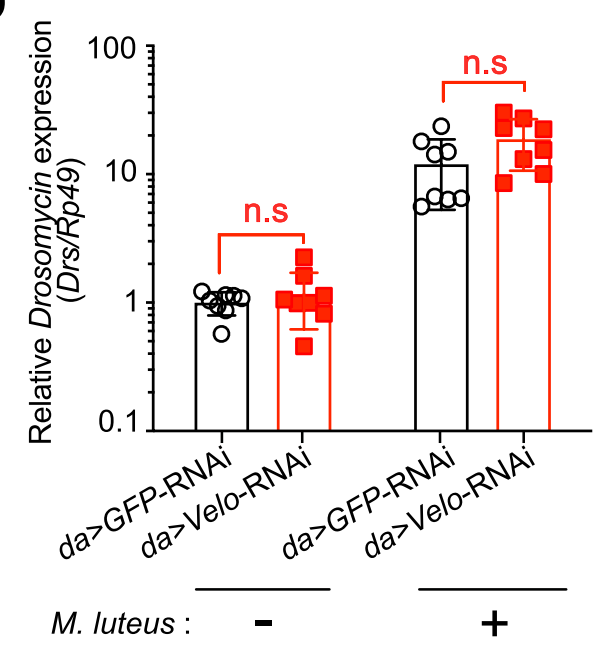

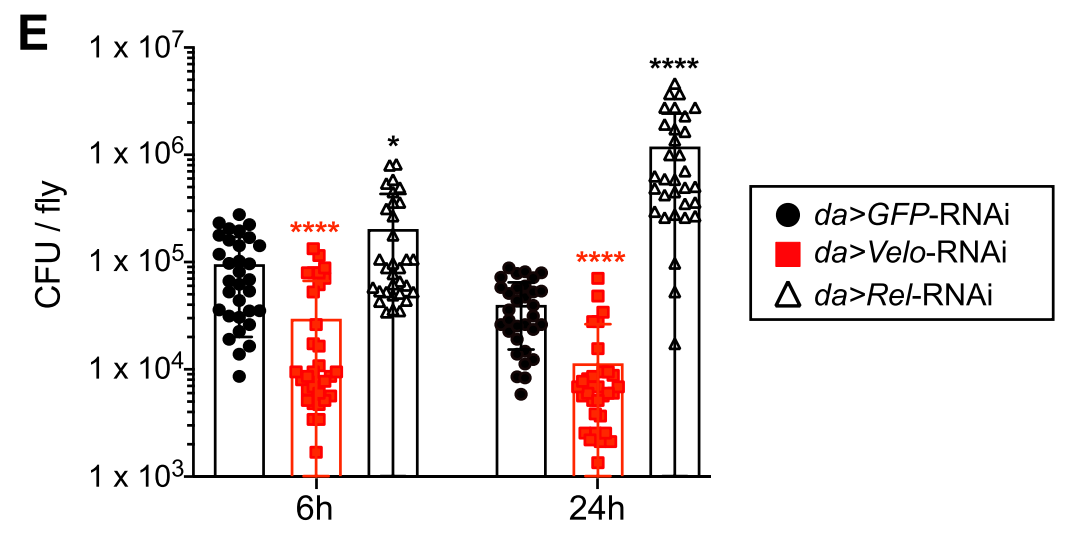

Figure 4. Ubiquitous knock-down of Velo results in shortened lifespan, elevated Diptericin expression, and decreased injected E. coli bacterial load in adult flies. (A) Ubiquitous Velo knock-down flies were generated by crossing UAS-Velo-RNAi with daughterless(da)-GAL4 line at $25^{\circ} \mathrm{C}$ (da>Velo-RNAi). The knock-down efficiency of Velo expression was examined by RT-qPCR. $d a>$ GFP-RNAi flies were used as a negative control. Expression of the Ribosomal protein 49 (Rp49) was used as the internal control for normalization. (B) The lifespan of $d a>$ Velo-RNAi flies was analyzed by counting the numbers of surviving flies daily at $25^{\circ} \mathrm{C}$. Data represent the mean with at least six biological replicates. The log-rank test was used to calculate the significance of survival curves for statistical analysis. (C,D) Three to five days after the eclosion, da > Velo-RNAi flies were pricked either with E. coli or M. luteus and the expression of Diptericin (C) or Drosomycin (D) were monitored at $6 \mathrm{~h}$ or $24 \mathrm{~h}$ post-infection, respectively. $d a>d I K K \beta-R N A i$ flies were used as a positive control. (E) $d a>V e l o-R N A i$ flies were infected by kanamycin-resistant $E$. coli, and the bacterial load was monitored at indicated time points. The data represent the mean and standard error of three independent experiments, and one data point represents a pool of 5-8 flies. $d a>$ Rel-RNAi flies were used as a positive control. The difference between control GFP and each target RNAi is statistically significant (student's $t$ test: ${ }^{\star} p<0.05,{ }^{\star * * *} p<0.0001$ ). 
bassiana (Fig. 5H). These results confirmed that Velo negatively regulates the IMD pathway at major immune organs, namely the fat body in adult flies.

\section{Discussion}

Verloren (Velo) means "Loss" in Germany as this gene was originally named after the lethal phenotype with a severe loss of neuronal pathfinding in embryos ${ }^{57}$. Indeed, previous RNAi screens had scored Velo as an essential gene involved in various cellular processes and signaling pathways such as (1) cell growth and viability ${ }^{59},(2)$ stem $^{2}$ cell maintenance ${ }^{60},(3)$ Notch pathway regulation ${ }^{61,62},(4)$ Wnt signaling $^{63}$, etc. These data indicate that Velo is involved in diverse developmental processes. Consistently, we observed that knock-down of Velo in flies led to a partial lethality at larval/pupal stage (with da-Gal4 driver), reduced lifespan (with both da-Gal4 and c564-Gal4 drivers) and high susceptibility to P. aeruginosa infection. Nonetheless, both $d a>$ Velo-RNAi and $c 564>$ VeloRNAi flies showed the upregulation of the IMD pathway dependent AMP expression. Given the facts that fly mutants for negative regulators of the IMD pathway are reported to have a short lifespan and some displayed high susceptibility to bacterial infections $s^{40,64,65}$, our result suggests that the high lethality of $c 564>$ Velo-RNAi flies is due to an excess activation of the IMD pathway upon bacterial infection. Importantly, this AMP upregulation was also observed in S2 cells specific to the IMD pathway but not to the Toll pathway. In this context, our data is the first demonstration that Velo is involved not only in development but also in the innate immune response by negative regulation of the IMD pathway.

To address where Velo acts in the IMD pathway, we performed two experiments, namely double knock-down and overexpression in S2 cells. As shown in Fig. 2, we observed significantly decreased Attacin-luciferase activity in the double knock-down condition, but the opposite phenotype was not noted in the overexpression analyses. We also did not observe the interaction between Velo and PGRP-LC in our co-immunoprecipitation assay. By these results, it was difficult to position Velo in the IMD pathway. Interestingly, we obtained a stimulating result from our immune cell staining experiment that Velo localizes, both in the nucleus and cytoplasm, but the majority is located in the nucleus (Fig. 3B,C). Indeed, protein sequence annotation database Pantree ${ }^{66}$ predicted that CG10107 (unannotated gene before naming as Velo) is localized in both the nucleus and cytoplasm (ID number: PTN002930416). In the following studies, Berdnik et al. observed in their transgenic flies that a long-form of Velo is localized in the nucleus, proposing that Velo might act as SUMO protease in the nucleus ${ }^{57}$. Dr. Cavalli's group elegantly discovered that Velo is required for the deSUMOylation of epigenetic repressor Polycomb (Pc) protein and changing the distribution and binding of PcG proteins to their chromatin targeting sites in the nucleus ${ }^{67}$. Interestingly, the Drosophila homolog of the yeast SWI2/SFN2 gene, Brahma (Brm), was isolated as a dominant suppressor of Pc mutations ${ }^{68}$ and reported to be a co-activator of trithorax group (trxG) protein Zeste ${ }^{69}$. This Brahma complex together with a novel nuclear factor Akirin was shown to be required for the transcription of a subset of effector genes in the IMD pathway ${ }^{70}$.

In mammals, there are at least two different isoforms of SENP7 (the closest homolog to Velo), namely long (SENP7L) and short (SENP7S). SENP7L resides mainly in the nucleus, whereas SENP7S is exclusively localized in the cytosol ${ }^{71}$. SENP7L contains a conserved heterochromatin protein 1 homolog (HP1)-box (PxVxL) motif which determines the mutual recruitment of SENP7 and HP1 $\alpha$ to heterochromatin ${ }^{72,73}$. In contrast, SENP7S lacks this HP-1-binding domain, explaining its cytosolic distribution ${ }^{71}$. Interestingly, the short-form of Velo contains one PxVxL motif at the amino acid 89th position, whereas the long-form contains two motifs at the 1068th and 1211th positions. Coherent to this, our immune cell staining result showed that the short-form of Velo has more cytoplasmic diffusion compared to the long-form (Fig. 3C). It is also interesting to note that Studencka et al. reported that HP1 and linker histone is required for the regulation of innate immune gene expression in $C$. elegans $^{74}$. Velo-mediated modulation of chromatin remodeling factors and identification of the cytosolic partners will be one of our future investigations.

Taken together, our data indicated that Velo is a new negative regulator of the IMD pathway both in vitro and in vivo systems. We suspect that due to some technical limitations, we cannot address some critical questions yet, like what are the targets of Velos in the nucleus and/or the cytoplasm, what if nuclear Velo affects chromatin remodeling or probably modulates Akirin-mediated NF- $\kappa$ B signaling ${ }^{58,70,75,76}$, how Velo contributes to SUMOylation status of the IMD pathway, etc. Further analyses will be required to understand the Velo-mediated negative regulation mechanism in the IMD pathway. 
A

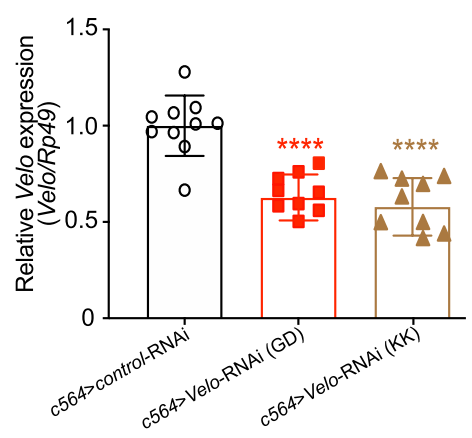

C

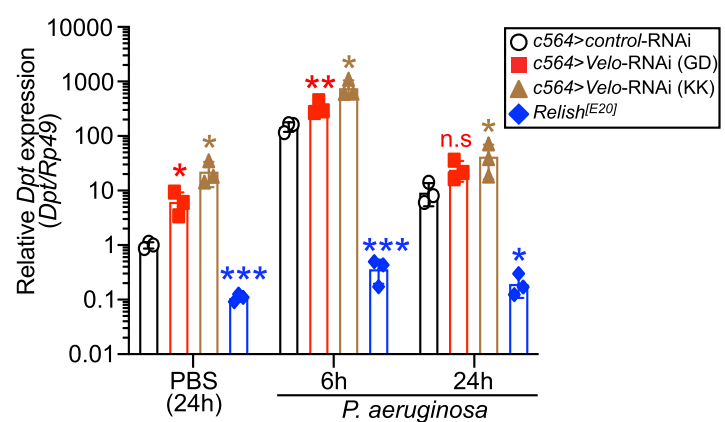

E
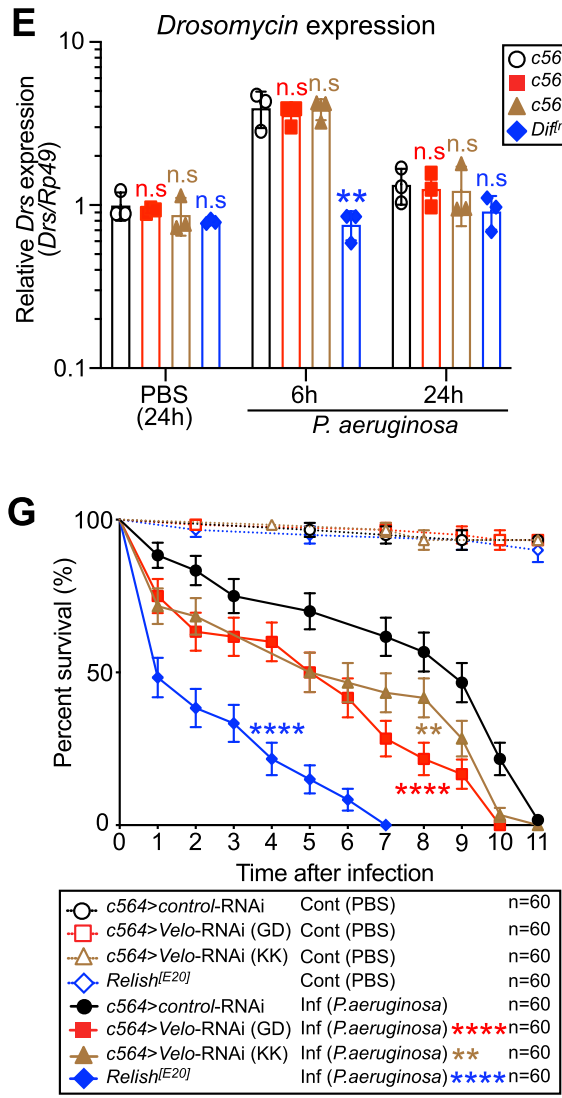
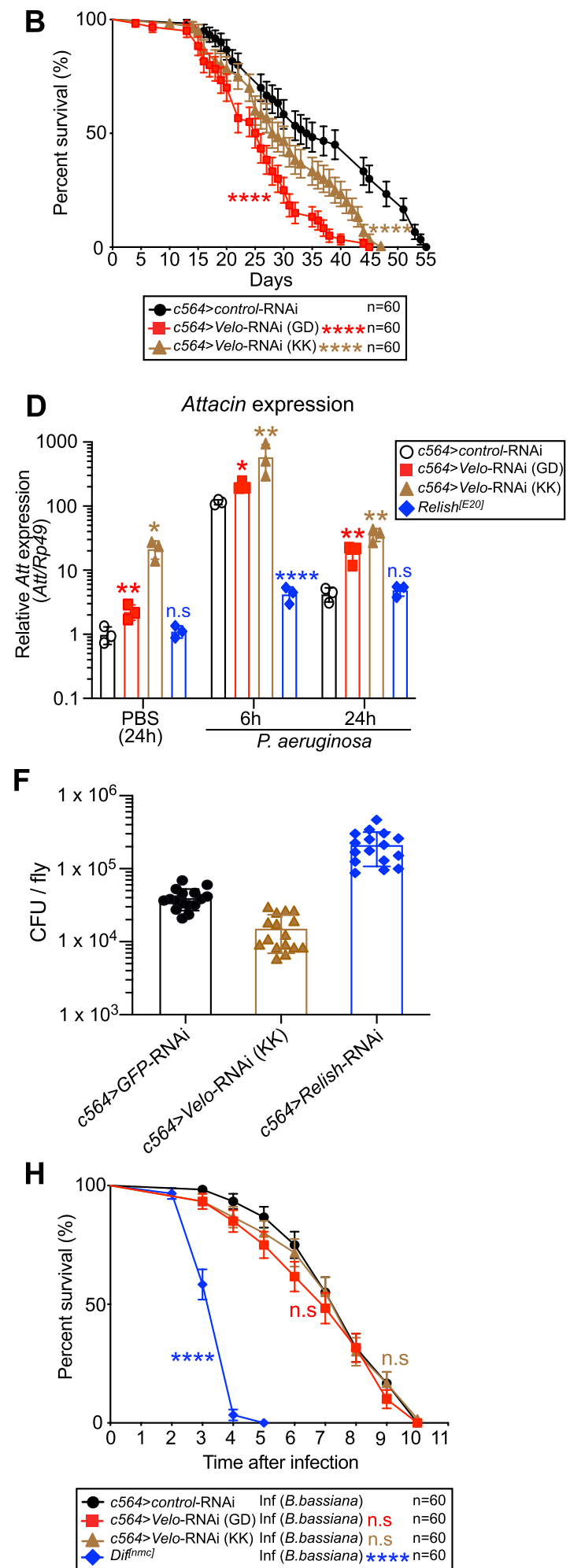
4Figure 5. Fat body-specific Velo knock-down flies show a moderate lifespan defect, elevated expression of the IMD pathway controlled AMPs, and high susceptibility to P. aeruginosa infection. (A) Two independent UAS-Velo-RNAi flies designated as (GD) and (KK) were crossed with c564-GAL4 driver to generate the fat body-specific Velo knock-down flies ( $c 564>$ Velo-RNAi). The level of Velo expression was measured as compared to that of control flies ( $c 564>$ control-RNAi). Expression of the Ribosomal protein 49 (Rp49) was used as the internal control for normalization. (B) The lifespan of two independent $c 564>V e l o-R N A i$ lines, as well as its control-RNAi, was monitored by counting the numbers of surviving flies daily at $25^{\circ} \mathrm{C}$. (C-E) $c 564>$ Velo-RNAi flies were pricked by P. aeruginosa and the expression of Diptericin, Attacin, and Drosomycin were monitored at $6 \mathrm{~h}$ and $24 \mathrm{~h}$ post-infection. (F) Twenty-four hour after infection of $P$. aeruginosa, the bacterial burden on $c 564>$ Velo-RNA $i$ flies was monitored by CFU assay. The data represent the mean and standard error of three biological replicates, and one data point represents a pool of 6-8 flies. The difference between control-RNAi and each Velo-RNAi is statistically significant (student's $t$ test: ${ }^{*} p<0.05,{ }^{* *} p<0.01,{ }^{* *} p<0.001$. n.s. indicates statistically non-significant). (G,H) The survival of the flies after infection of $P$. aeruginosa $(\mathbf{G})$ or naturally with B. bassiana (H) was monitored. Control PBS buffer pricked flies were used as control. Relish ${ }^{[E 20]}$ or Dif ${ }^{[n m c]}$ null mutant flies were used as positive controls. Data represent the mean with at least six biological replicates. The log-rank test was used to calculate the significance of survival curves for statistical analysis.

\section{Data availability}

The data that support the findings of this study are available from the corresponding author, [A.G.], upon reasonable request.

Received: 12 February 2021; Accepted: 16 July 2021

Published online: 30 July 2021

\section{References}

1. Hoffmann, J. A. The immune response of Drosophila. Nature 426, 33-38 (2003).

2. Hoffmann, J. A. \& Reichhart, J.-M. Drosophila innate immunity: An evolutionary perspective. Nat. Immunol. 3, 121-126 (2002).

3. Hoffmann, J. A., Kafatos, F. C., Janeway, C. A. \& Ezekowitz, R. A. Phylogenetic perspectives in innate immunity. Science 284, 1313-1318 (1999).

4. Brennan, C. A. \& Anderson, K. V. Drosophila: The genetics of innate immune recognition and response. Annu. Rev. Immunol. 22, 457-483 (2004).

5. Akira, S., Uematsu, S. \& Takeuchi, O. Pathogen recognition and innate immunity. Cell 124, 783-801 (2006).

6. Bulet, P., Hetru, C., Dimarcq, J. L. \& Hoffmann, D. Antimicrobial peptides in insects; structure and function. Dev. Comp. Immunol. 23, 329-344 (1999).

7. Imler, J.-L. \& Bulet, P. Antimicrobial peptides in Drosophila: Structures, activities and gene regulation. In Chemical Immunology and Allergy (eds Kabelitz, D. \& Schröder, J.-M.) 1-21 (KARGER, 2005).

8. Boman, H. G. Antibacterial peptides: Basic facts and emerging concepts. J. Intern. Med. 254, 197-215 (2003).

9. Benfield, A. H. \& Henriques, S. T. Mode-of-action of antimicrobial peptides: Membrane disruption vs intracellular mechanisms. Front. Med. Technol. 2, 610997 (2020).

10. Hanson, M. A. \& Lemaitre, B. New insights on Drosophila antimicrobial peptide function in host defense and beyond. Curr. Opin. Immunol. 62, 22-30 (2020).

11. Lemaitre, B., Nicolas, E., Michaut, L., Reichhart, J. M. \& Hoffmann, J. A. The dorsoventral regulatory gene cassette spätzle/Toll/ cactus controls the potent antifungal response in Drosophila adults. Cell 86, 973-983 (1996).

12. Lemaitre, B. et al. A recessive mutation, immune deficiency (imd), defines two distinct control pathways in the Drosophila host defense. Proc. Natl. Acad. Sci. USA 92, 9465-9469 (1995).

13. Ferrandon, D., Imler, J. L., Hetru, C. \& Hoffmann, J. A. The Drosophila systemic immune response: Sensing and signalling during bacterial and fungal infections. Nat. Rev. Immunol. 7, 862-874 (2007).

14. Lemaitre, B. \& Hoffmann, J. The host defense of Drosophila melanogaster. Annu. Rev. Immunol. 25, 697-743 (2007).

15. Tauszig-Delamasure, S., Bilak, H., Capovilla, M., Hoffmann, J. A. \& Imler, J.-L. Drosophila MyD88 is required for the response to fungal and Gram-positive bacterial infections. Nat. Immunol. 3, 91-97 (2002).

16. Sun, H., Bristow, B. N., Qu, G. \& Wasserman, S. A. A heterotrimeric death domain complex in Toll signaling. Proc. Natl. Acad. Sci. USA 99, 12871-12876 (2002).

17. Rutschmann, S. et al. The Rel protein DIF mediates the antifungal but not the antibacterial host defense in Drosophila. Immunity 12, 569-580 (2000).

18. Gottar, M. et al. Dual detection of fungal infections in Drosophila via recognition of glucans and sensing of virulence factors. Cell 127, 1425-1437 (2006).

19. Gobert, V. et al. Dual activation of the Drosophila toll pathway by two pattern recognition receptors. Science 302, 2126-2130 (2003).

20. Michel, T., Reichhart, J. M., Hoffmann, J. A. \& Royet, J. Drosophila Toll is activated by Gram-positive bacteria through a circulating peptidoglycan recognition protein. Nature 414, 756-759 (2001)

21. Ligoxygakis, P., Pelte, N., Hoffmann, J. A. \& Reichhart, J.-M. Activation of Drosophila Toll during fungal infection by a blood serine protease. Science 297, 114-116 (2002).

22. Issa, N. et al. The circulating protease persephone is an immune sensor for microbial proteolytic activities upstream of the Drosophila toll pathway. Mol. Cell 69, 539-550.e6 (2018).

23. Kaneko, T. et al. Monomeric and polymeric gram-negative peptidoglycan but not purified LPS stimulate the Drosophila IMD pathway. Immunity 20, 637-649 (2004).

24. Kaneko, T. et al. PGRP-LC and PGRP-LE have essential yet distinct functions in the drosophila immune response to monomeric DAP-type peptidoglycan. Nat. Immunol. 7, 715-723 (2006).

25. Takehana, A. et al. Peptidoglycan recognition protein (PGRP)-LE and PGRP-LC act synergistically in Drosophila immunity. EMBO J. 23, 4690-4700 (2004).

26. Georgel, P. et al. Drosophila immune deficiency (IMD) is a death domain protein that activates antibacterial defense and can promote apoptosis. Dev. Cell 1, 503-514 (2001).

27. Naitza, S. et al. The Drosophila immune defense against gram-negative infection requires the death protein dFADD. Immunity 17, 575-581 (2002). 
28. Leulier, F., Vidal, S., Saigo, K., Ueda, R. \& Lemaitre, B. Inducible expression of double-stranded RNA reveals a role for dFADD in the regulation of the antibacterial response in Drosophila adults. Curr. Biol. 12, 996-1000 (2002).

29. Leulier, F., Rodriguez, A., Khush, R. S., Abrams, J. M. \& Lemaitre, B. The Drosophila caspase Dredd is required to resist gramnegative bacterial infection. EMBO Rep. 1, 353-358 (2000).

30. Gesellchen, V., Kuttenkeuler, D., Steckel, M., Pelte, N. \& Boutros, M. An RNA interference screen identifies inhibitor of apoptosis protein 2 as a regulator of innate immune signalling in Drosophila. EMBO Rep. 6, 979-984 (2005).

31. Kleino, A. et al. Inhibitor of apoptosis 2 and TAK1-binding protein are components of the Drosophila Imd pathway. EMBO J. 24, 3423-3434 (2005).

32. Leulier, F., Lhocine, N., Lemaitre, B. \& Meier, P. The Drosophila inhibitor of apoptosis protein DIAP2 functions in innate immunity and is essential to resist gram-negative bacterial infection. Mol. Cell. Biol. 26, 7821-7831 (2006).

33. Vidal, S. et al. Mutations in the Drosophila dTAK1 gene reveal a conserved function for MAPKKKs in the control of rel/NFkappaB-dependent innate immune responses. Genes Dev. 15, 1900-1912 (2001).

34. Silverman, N. et al. A Drosophila IkappaB kinase complex required for Relish cleavage and antibacterial immunity. Genes Dev. 14, 2461-2471 (2000).

35. Rutschmann, S. et al. Role of Drosophila IKK gamma in a toll-independent antibacterial immune response. Nat. Immunol. 1, 342-347 (2000).

36. Stoven, S. et al. Caspase-mediated processing of the Drosophila NF-kappaB factor Relish. Proc. Natl. Acad. Sci. USA 100, 5991-5996 (2003).

37. Stöven, S., Ando, I., Kadalayil, L., Engström, Y. \& Hultmark, D. Activation of the Drosophila NF-kappaB factor Relish by rapid endoproteolytic cleavage. EMBO Rep. 1, 347-352 (2000).

38. Lee, K. Z. \& Ferrandon, D. Negative regulation of immune responses on the fly. EMBO J. 30, 988-990 (2011)

39. Myllymaki, H., Valanne, S. \& Ramet, M. The Drosophila imd signaling pathway. J. Immunol. 192, 3455-3462 (2014).

40. Paredes, J. C., Welchman, D. P., Poidevin, M. \& Lemaitre, B. Negative regulation by amidase PGRPs shapes the Drosophila antibacterial response and protects the fly from innocuous infection. Immunity 35, 770-779 (2011).

41. Bischoff, V. et al. Downregulation of the Drosophila immune response by peptidoglycan-recognition proteins SC1 and SC2. PLoS Pathog. 2, e14 (2006).

42. Basbous, N. et al. The Drosophila peptidoglycan-recognition protein LF interacts with peptidoglycan-recognition protein LC to downregulate the Imd pathway. EMBO Rep. 12, 327-333 (2011).

43. Kleino, A. et al. Pirk is a negative regulator of the Drosophila Imd pathway. J. Immunol. 180, 5413-5422 (2008).

44. Aggarwal, K. et al. Rudra interrupts receptor signaling complexes to negatively regulate the IMD pathway. PLoS Pathog. 4, e1000120 (2008).

45. Lhocine, N. et al. PIMS modulates immune tolerance by negatively regulating Drosophila innate immune signaling. Cell Host Microbe 4, 147-158 (2008).

46. Ragab, A. et al. Drosophila Ras/MAPK signalling regulates innate immune responses in immune and intestinal stem cells. EMBO J. 30, 1123-1136 (2011).

47. Thevenon, D. et al. The Drosophila ubiquitin-specific protease dUSP36/Scny targets IMD to prevent constitutive immune signaling. Cell Host Microbe 6, 309-320 (2009).

48. Yagi, Y., Lim, Y. M., Tsuda, L. \& Nishida, Y. fat facets induces polyubiquitination of Imd and inhibits the innate immune response in Drosophila. Genes Cells 18, 934-945 (2013).

49. Tsuda, M., Langmann, C., Harden, N. \& Aigaki, T. The RING-finger scaffold protein Plenty of SH3s targets TAK1 to control immunity signalling in Drosophila. EMBO Rep. 6, 1082-1087 (2005).

50. Fernando, M. D. A., Kounatidis, I. \& Ligoxygakis, P. Loss of Trabid, a new negative regulator of the drosophila immune-deficiency pathway at the level of TAK1, reduces life span. PLoS Genet. 10, 1004117 (2014).

51. Trompouki, E. et al. CYLD is a deubiquitinating enzyme that negatively regulates NF-kappaB activation by TNFR family members. Nature 424, 793-796 (2003).

52. Tsichritzis, T. et al. A Drosophila ortholog of the human cylindromatosis tumor suppressor gene regulates triglyceride content and antibacterial defense. Development 134, 2605-2614 (2007).

53. Aparicio, R., Neyen, C., Lemaitre, B. \& Busturia, A. dRYBP contributes to the negative regulation of the Drosophila Imd pathway. PLoS One 8, e62052 (2013).

54. Fukuyama, H. et al. Landscape of protein-protein interactions in Drosophila immune deficiency signaling during bacterial challenge. Proc. Natl. Acad. Sci. USA 110, 10717-10722 (2013).

55. Kenmoku, H., Hori, A., Kuraishi, T. \& Kurata, S. A novel mode of induction of the humoral innate immune response in Drosophila larvae. Dis. Model. Mech. 10, 271-281 (2017).

56. Liberati, N. T. et al. An ordered, nonredundant library of Pseudomonas aeruginosa strain PA14 transposon insertion mutants. Proc. Natl. Acad. Sci. USA 103, 2833-2838 (2006).

57. Berdnik, D., Favaloro, V. \& Luo, L. The SUMO protease Verloren regulates dendrite and axon targeting in olfactory projection neurons. J. Neurosci. 32, 8331-8340 (2012).

58. Goto, A. et al. Akirins are highly conserved nuclear proteins required for NF-kappaB-dependent gene expression in drosophila and mice. Nat. Immunol. 9, 97-104 (2008).

59. Boutros, M. et al. Genome-wide RNAi analysis of growth and viability in Drosophila cells. Science 303, 832-835 (2004).

60. Sanchez, C. G. et al. Regulation of ribosome biogenesis and protein synthesis controls germline stem cell differentiation. Cell Stem Cell 18, 276-290 (2016).

61. Saj, A. et al. A combined ex vivo and in vivo RNAi screen for notch regulators in Drosophila reveals an extensive notch interaction network. Dev. Cell 18, 862-876 (2010).

62. Mummery-Widmer, J. L. et al. Genome-wide analysis of Notch signalling in Drosophila by transgenic RNAi. Nature 458, 987-992 (2009).

63. DasGupta, R., Kaykas, A., Moon, R. T. \& Perrimon, N. Functional genomic analysis of the Wnt-wingless signaling pathway. Science 308, 826-833 (2005).

64. Costechareyre, D. et al. Tissue-specific regulation of drosophila NF-x03BA;B pathway activation by peptidoglycan recognition protein SC. J. Innate Immun. 8, 67-80 (2016).

65. Ryu, J.-H. et al. Innate immune homeostasis by the homeobox gene caudal and commensal-gut mutualism in Drosophila. Science 319, 777-782 (2008)

66. Gaudet, P., Livstone, M. S., Lewis, S. E. \& Thomas, P. D. Phylogenetic-based propagation of functional annotations within the Gene Ontology consortium. Brief. Bioinform. 12, 449-462 (2011).

67. Gonzalez, I., Mateos-Langerak, J., Thomas, A., Cheutin, T. \& Cavalli, G. Identification of regulators of the three-dimensional polycomb organization by a microscopy-based genome-wide RNAi screen. Mol Cell 54, 485-499 (2014).

68. Kennison, J. A. \& Tamkun, J. W. Dosage-dependent modifiers of polycomb and antennapedia mutations in Drosophila. Proc. Natl. Acad. Sci. USA 85, 8136-8140 (1988).

69. Kal, A. J., Mahmoudi, T., Zak, N. B. \& Verrijzer, C. P. The Drosophila brahma complex is an essential coactivator for the trithorax group protein zeste. Genes Dev. 14, 1058-1071 (2000). 
70. Bonnay, F. et al. Akirin specifies NF-kappaB selectivity of Drosophila innate immune response via chromatin remodeling. EMBO J. 33, 2349-2362 (2014).

71. Bawa-Khalfe, T. et al. Differential expression of SUMO-specific protease 7 variants regulates epithelial-mesenchymal transition. Proc. Natl. Acad. Sci. USA 109, 17466-17471 (2012).

72. Maison, C. et al. The SUMO protease SENP7 is a critical component to ensure HP1 enrichment at pericentric heterochromatin. Nat. Struct. Mol. Biol. 19, 458-460 (2012).

73. Romeo, K. et al. The SENP7 SUMO-protease presents a module of two HP1 interaction motifs that locks HP1 protein at pericentric heterochromatin. Cell Rep. 10, 771-782 (2015).

74. Studencka, M. et al. Novel roles of Caenorhabditis elegans heterochromatin protein HP1 and linker histone in the regulation of innate immune gene expression. Mol. Cell. Biol. 32, 251-265 (2012).

75. Cammarata-Mouchtouris, A. et al. Hyd ubiquitinates the NF- $\mathrm{kB}$ co-factor Akirin to operate an effective immune response in Drosophila. PLoS Pathog. 16, el008458 (2020).

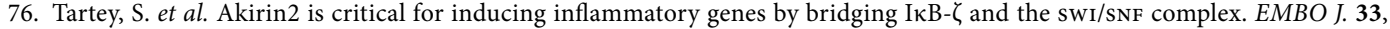
2332-2348 (2014).

\section{Acknowledgements}

We thank Audrey Melignon for excellent technical assistance, Pr. Jules Hoffmann, Pr. Jean-Luc Imler, Dr. Dominique Ferrandon, and Dr. João Marques for critical reading of the manuscript and insightful suggestions. Pr. Liqun Luo for pAWH-VeloWT and -VeloCS plasmids. Dr. Takayuki Kuraishi and Pr. Shoichiro Kurata for $D^{f} f^{n m c}$ mutant. Bloomington and VDRC Stock Centers for fly strains. This work was supported by the National Institutes of Health (PO1 AI070167), the Balzan Foundation (to J.A.H.), the Investissement d'Avenir Programs (ANR-11-EQPX-0022), the Sino-French Hoffmann Institute, LIA (Laboratoire international associé) «REL2 and resistance to malaria», USIAS (University of Strasbourg Institute for Advanced Study), CNRS, and INSERM.

\section{Author contributions}

P.P., A.R., and A.G. performed experiments; P.P. and A.G. designed the experiments and analyzed the data; P.P. and A.G. wrote the manuscript and prepared figures. All authors reviewed the manuscript.

\section{Competing interests}

The authors declare no competing interests.

\section{Additional information}

Supplementary Information The online version contains supplementary material available at https://doi.org/ 10.1038/s41598-021-94973-0.

Correspondence and requests for materials should be addressed to A.G.

Reprints and permissions information is available at www.nature.com/reprints.

Publisher's note Springer Nature remains neutral with regard to jurisdictional claims in published maps and institutional affiliations.

(c) (i)

Open Access This article is licensed under a Creative Commons Attribution 4.0 International License, which permits use, sharing, adaptation, distribution and reproduction in any medium or format, as long as you give appropriate credit to the original author(s) and the source, provide a link to the Creative Commons licence, and indicate if changes were made. The images or other third party material in this article are included in the article's Creative Commons licence, unless indicated otherwise in a credit line to the material. If material is not included in the article's Creative Commons licence and your intended use is not permitted by statutory regulation or exceeds the permitted use, you will need to obtain permission directly from the copyright holder. To view a copy of this licence, visit http://creativecommons.org/licenses/by/4.0/.

(C) The Author(s) 2021 